\title{
Triggers of suicide ideation and protective factors of actually executing suicide among first onset cases in older psychiatric outpatients: a qualitative study
}

Shwu-Hua Lee ${ }^{1,2}$, Yun-Fang Tsai ${ }^{3,4^{*}}$, Ching-Yen Chen ${ }^{1}$ and Li-Bi Huang ${ }^{5}$

\begin{abstract}
Background: Suicide is a global issue among the elderly, but few studies have explored the experiences of suicide ideation in older Asian psychiatric outpatients.

Method: Older psychiatric outpatients $(N=24)$ were recruited by convenience from one medical centre and one regional hospital in northern Taiwan. Participants were recruited if they met these inclusion criteria: 1) $\geq 65$ years old, 2) without severe cognitive deficit, 3) outpatients in the psychiatric clinics at the selected hospitals, and 4) self-reported first episode of suicidal ideation within the previous year. Data were collected in individual interviews using a semi-structured guide and analysed by content analysis.

Results: Suicide ideation was triggered by illness and physical discomfort, conflicts with family members/friends, illness of family members, death of family members/friends, and loneliness. Participants' reasons for not executing suicide were family members' and friends' support, receiving treatment, finding a way to shift their attention, fear of increasing pressure on one's children, religious beliefs, and not knowing how to execute suicide.

Conclusion: Understanding these identified triggers of suicide ideation may help psychiatrists open a channel for conversation with their elderly clients and more readily make their diagnosis. Understanding these identified protective factors against executing suicide can help psychiatrists not only treat depression, but also enhance protective factors for their clients.
\end{abstract}

Keywords: Suicide ideation, Psychiatric, Outpatients, Older people

\section{Background}

Suicide is a global issue among older people [1]. The most significant predictor of suicide is depression for older people [2,3]. Therefore, depression screening and treatment is commonly suggested for suicide prevention among older people [4]. Suicidal ideation in over 21,000 older Australian community-dwelling adults was independently associated with male gender, higher education, current smoking, living alone, poor social support, no religious practice, financial strain, childhood physical abuse, history

\footnotetext{
* Correspondence: yftsai@mail.cgu.edu.tw

${ }^{3}$ School of Nursing, College of Medicine, Chang Gung University, 259

Wen-Hwa 1st Road, Kwei-Shan, Tao-Yuan, Taiwan

${ }^{4}$ Department of Nursing, Chang Gung Memorial Hospital at Keelung, 222

Maijin Road, Keelung, Taiwan

Full list of author information is available at the end of the article
}

of familial suicide, past depression, current anxiety, depression or comorbid anxiety and depression, past suicide attempt, pain, poor self-perceived health and current use of antidepressants [5]. Similarly, a current wish to die in a population-based study of both community-dwelling and institutionalised older Dutch adults was associated with depressive symptoms, depressive disorder, lower perceived mastery, financial problems, loneliness, small social network, involuntary urine loss, being divorced, and speech impairment [6]. In 665 older community-dwelling adults from Hong Kong, suicidal ideation was predicted by poor vision, hearing problems, more diseases, and depressive symptoms [7]. Other factors related to older adults' suicide ideation were insomnia symptoms [8], functional impairment [9], and perceived burden to others [9]. Furthermore, 
a wish to die was found in a qualitative study of older Dutch adults to either be triggered suddenly after a traumatic life event or developed slowly after a lifetime of difficulties, because of aging or illness, or with recurring depression [10]. These older people considered their situation unacceptable and out of their control to change. Therefore, they gradually gave up to trying to cope and began developing a perspective of death as something positive or a release from the situation; such thoughts were perceived as a way for them to reclaim control [10].

In suicide research, whether one attempts or completes suicide is related to one's beliefs and hopeless expectancies [11]. Since beliefs and expectations about suicidal behaviours in older people with suicide ideation may be considered protective factors against suicide, they are important to understand. Understanding these factors can help psychiatrists not only to treat depression, but also to enhance protective factors (beliefs and expectations). Some protective factors against suicide identified in older people include sense of belonging [12,13], reasons for living [12], and perceived social support [14].

Most studies on suicide among older people have been conducted in western populations, with few studies in Asia. Moreover, these few Asia-based suicide studies explored older people who lived in institutions $[15,16]$ or in the community as non-psychiatric patients [17-19]. Since suicidal ideation is a risk factor for attempted and completed suicide [20], early detection and treatment of suicidal ideation is important. Unfortunately, few studies have explored newonset experiences of suicidal ideation among older community-dwelling psychiatric patients, particularly those with depression, either in the West or in Asia. Among older community-dwelling psychiatric patients in the West, factors related to suicide ideation include neurotic personality [21], hopelessness [22], responsibility to family [22], and lower quality of life [23]. Protective factors against suicidal ideation in Western populations of psychiatric outpatients included fear of suicide [22] and family connectedness [24]. As the epidemiology of suicide varies in different cultures, studies conducted in the West may not be generalisable to Asia [25]. To address these gaps in the literature, this study aimed to explore triggers of suicide ideation among older first onset cases in psychiatric outpatients in Taiwan and their reasons for not executing suicide.

\section{Method}

\section{Design}

This qualitative descriptive study was part of a large research series to develop a suicide-prevention model for older people. Data were collected by individual interviews.

\section{Sample and setting}

We recruited elderly psychiatric outpatients by convenience sampling from one medical centre and one regional hospital in northern Taiwan. Participants were recruited if they met these inclusion criteria: 1) $\geq 65$ years old, 2) without severe cognitive deficit (Chinese version MiniMental State Examination [MMSE] score $\geq 16$ for those without formal education; MMSE score $\geq 20$ for primary school graduates or above [26], 3) outpatients in the psychiatric clinics at the selected hospitals, and 4) selfreported first episode of suicidal ideation within the previous year. Participants were excluded if they had made a previous suicide attempt. The reason for limiting suicidal ideation to within 1 year was to avoid participants' memory bias. Elderly outpatients who met these criteria were referred by their clinical psychiatrists since they are responsible for assessing and documenting their patients' health information. These clinical psychiatrists regularly used the MINI International Neuropsychiatric Interview [27] which was translated into Mandarin by the Taiwanese Society of Psychiatry to diagnose clients.

\section{Data collection}

Data were collected by a trained research assistant (RA, see training details below) in 24 audiotaped individual interviews, each lasting 30 to 60 minutes. Interviews were conducted in Mandarin Chinese in a private room at the selected hospitals. The RA encouraged participants to share their experiences by using a semistructured interview guideline (e.g. 'How and when did you start to have suicidal thoughts? Please describe your life condition around that period. What were the reasons for you to have these thoughts (about suicide)? What were the reasons for your not actually executing suicide?') Participants' personal characteristics and illness-related information were also collected by a structured form. Immediately after each interview, the RA used memos and a reflective journal to record observations about participants' behaviour during interviews and ideas about coding, respectively. Data were collected until analysis showed no new themes (data saturation).

The RA was a master's-prepared psychiatric nurse with 14 years experience in caring for psychiatric patients. Moreover, she had worked more than 6 years with our research team on our series of studies on older people's depression and suicide. For this study, she attended at least three training sessions under the supervision of the second author. In the first session, the second author explained the study purpose, design, and content. In the second session, the RA practiced using the interview guide with two patients until she became familiar with it. In the third session, the RA practiced observing non-verbal behaviours and taking notes after practice interviews with another two patients. 


\section{Ethical considerations}

This study was approved by the Institutional Review Board (IRB) of Chang Gung Medical Foundation. After the IRB approved the study, the RA approached older outpatients referred by their physicians. The RA described the study purpose and procedure to outpatients, the required time commitment, confidentiality, and their right not to participate or to withdraw from the study at any time, and obtained their written consent to participate. Older outpatients received no compensation for their participation.

\section{Data analysis}

All audiotapes were transcribed verbatim (in Mandarin) as soon as interviews ended. Transcripts were first compared with audiotapes for accuracy, and relevant information such as emotional content and nonverbal behaviour was noted from memos and the reflective journal. All interview transcripts were then analysed by content analysis to reveal themes in the interview data. The transcripts were coded individually by all authors, who then compared and discussed themes and codes. Discrepancies were resolved by discussion to reach agreement. Content analysis was selected for data analysis because it can be used to characterise the content of recorded communication [28]. Each theme was considered formed when at least $20 \%$ of participants expressed a similar concept.

\section{Rigour}

Trustworthiness of the data was established by authors' prolonged engagement with transcripts, methodological triangulation, participant observation, peer debriefing, and reflective journaling [29]. Method triangulation involved comparing data from transcripts and from other sources (memos, reflective journal, and debriefing notes). Memos on participant observation were kept by the RA. For peer debriefing, all authors discussed the analysis with experts in qualitative research. To ensure confirmability, the second author made memos and kept a reflective journal on the decisions made throughout the study, thus allowing audit. Finally, reflexivity was promoted by all authors examining their own positions, beliefs and values and their effects on the research. These effects were minimised by the second author's reflexive journal and discussion among multiple authors.

\section{Results}

\section{Participants' characteristics and illness-related} information

Of 26 older psychiatric outpatients who met the criteria and were approached, two refused to participate. One did not want to be audiotaped and the other did not have time. The remaining 24 participants were on average 72.1 years old $(\mathrm{SD}=6.2$, range $=65-84)$. The majority was female (70.8\%), had graduated from a primary school or below (66.6\%), and was married (58.3\%). For details, see Table 1. Regarding clinical characteristics, most participants $(91.7 \%)$ were diagnosed with depressive disorder, and the rest (8.3\%) were diagnosed with anxiety disorder. Participants' average score on the Geriatric Depression Scale short-form [30] at the time of the interview was 6.6 $(\mathrm{SD}=4.2$, range $=1-13$ ), with $58.3 \%$ of them scoring $\geq 5$ to indicate a depressive tendency. Information about their chronic diseases can be found in Table 2 . The majority of participants $(75.0 \%)$ were brought to the clinics by their family members, $16.7 \%$ had been diagnosed with depressive disorder and had discontinued treatments but were having suicidal ideations for the first time, and $8.3 \%$ had been referred to the psychiatric clinics by their medicalsurgical physicians. At the time of interview for this study, participants had been having suicidal ideations on average 5.2 months $(\mathrm{SD}=3.7$, range $=0.5-12.0)$.

\section{Reasons for suicidal thoughts}

Analysis of interview data indicated that participants' reasons for suicidal ideation were related to five themes: illness and physical discomfort (58.3\%), conflicts with family members/friends (41.7\%), illness of family members

Table 1 Demographic characteristics of participating psychiatric outpatients $(N=24)$

\begin{tabular}{|c|c|c|c|c|}
\hline Characteristic & $n$ & $\%$ & Mean & SD \\
\hline Age (years) & & & 72.1 & 6.2 \\
\hline \multicolumn{5}{|l|}{ Gender } \\
\hline Male & 7 & 29.2 & & \\
\hline Female & 17 & 70.8 & & \\
\hline \multicolumn{5}{|l|}{ Formal education } \\
\hline None & 5 & 20.8 & & \\
\hline Primary School & 11 & 45.8 & & \\
\hline$\geq$ Junior High School & 8 & 33.3 & & \\
\hline \multicolumn{5}{|l|}{ Marital Status } \\
\hline Married & 14 & 58.3 & & \\
\hline Widow/widower & 8 & 37.5 & & \\
\hline Divorced & 1 & 4.2 & & \\
\hline \multicolumn{5}{|l|}{ Religious Beliefs } \\
\hline General folk beliefs & 9 & 37.5 & & \\
\hline Buddhism & 6 & 25.0 & & \\
\hline None & 5 & 20.8 & & \\
\hline Other & 4 & 16.7 & & \\
\hline \multicolumn{5}{|l|}{ Living Status } \\
\hline Living with spouse and children & 10 & 41.7 & & \\
\hline Living with children & 8 & 33.3 & & \\
\hline Living with spouse & 3 & 12.5 & & \\
\hline Living alone & 3 & 12.5 & & \\
\hline
\end{tabular}


Table 2 Chronic diseases in participating psychiatric outpatients $(N=24)$

\begin{tabular}{ll}
\hline Chronic disease & $\boldsymbol{n}(\%)$ \\
\hline Hypertension & $9(35.7)$ \\
Digestive system disease & $8(33.3)$ \\
Arthritis & $3(12.5)$ \\
Fracture & $2(8.3)$ \\
Cataract & $2(8.3)$ \\
Diabetes & $2(8.3)$ \\
Heart disease & $2(8.3)$ \\
Stroke & $2(8.3)$ \\
Urinary system disease & $1(4.2)$ \\
Cancer & $1(4.2)$ \\
Renal disease & $1(4.2)$ \\
Respiratory disease & $1(4.2)$ \\
\hline
\end{tabular}

(33.3\%), death of family members/friends (20.8\%), and loneliness (20.8\%). Among the participants, 37.5\% expressed only one of the five triggers, $50 \%$ expressed two of the five triggers, and $12.5 \%$ expressed three of the five triggers.

\section{Illness and physical discomfort}

Six participants said that they had suffered from chronic illness for a long time and wanted to be rid of it. As C68 said, 'My heart isn't good and I had a stroke last year. I can't control my hands well now [muscle weakness]. My heart disease has also bothered me for a long time. I can't breathe well. My life feels like an endless suffering. Death would get rid of it [the suffering].' Another trigger of suicidal ideation for two participants was having a chronic illness and suddenly being diagnosed with another one. As C3 said, 'I used to take medicine to control my hypertension. One day, the doctor said I have diabetes and need to control my sugar. My world suddenly turned black. When I thought of all the medicines I needed to take for the rest of my life, I felt worried and sad. Then I thought death might be a good way to resolve all of my health problems.'

In addition, two participants talked about feeling physical discomfort and consulting with several physicians. None of them could find the origin of their discomfort. Their mood became worse and they were transferred to psychiatric clinics. As C53 said, 'I felt heart palpitations and was sent to the ER by ambulance about 5 months ago. After I was discharged, I still felt very weak. I had visited several different clinics, such as cardiology, thyroid, and the emergency department; none of their physicians could tell me why I felt sick. I felt hopeless because my condition was getting worse. I kept losing weight. At that time, I started to have ideas [about suicide]. Because my brother-in-law was an old patient in this psychiatric clinic, he suggested that I see his psychiatrist. Eventually, I realised that I hadn't been sleeping well for about a year because I was under severe stress.'

Four participants expressed that their depression caused suicide ideation. As C43 said, 'At that time, I felt my life was a mess. I didn't know how I ought to take care of my children or my husband. I didn't know what I should cook or how to clean my home. Everything became very difficult for me. I was under great pressure. I knew I was sick [depressed] and couldn't stop thinking about death.' Similarly, C55 said, 'I just felt everything was wrong and it became easier to lose my temper. I felt depressed and kept having many negative thoughts. They [husband and son] took me to see the psychiatrist and I was told I had depression.'

\section{Conflicts with family members/friends}

Seven participants described feeling sad and angry because of conflicts with their adult children who did not take good care of them or respect them in their old age, despite the participants' lifelong efforts to raise their children. As C14 said, 'I worked very hard to save money to raise them. Now, my big [older] son shouts at me. My little [younger] son also shouts at me. Who am I?? How can they treat me like this? I couldn't find anyone to tell my story. I didn't want to tell my relatives or friends about my family situation. I don't have an outlet to share my feelings.' Three participants had conflicts with their friends. As C29 said, 'He was my friend. We planned to invest in a business together and had signed a contract with the seller. However, the seller suddenly broke the contract. It wasn't my fault. He [friend] started to refuse to talk to me. I felt upset and sad.'

\section{IIIness of family members}

Family members' illness had a great impact on eight participants' moods and even life. As C58 said, 'My husband had a second stroke. He is bed-ridden now. I need to take care of him. As a result, I don't eat and sleep well. I feel very anxious and stressed. We couldn't hire a person to help us because we didn't have enough money. When I felt very tired, I really thought about death.' Similarly, C45 said, 'My son was diagnosed with nasopharyngeal cancer. I couldn't accept it. How could it happen? I was so sad.'

\section{Death of family members/friends}

Family members and friends' deaths were another trigger for five participants' suicide ideation. As C42 said, 'My mood was so low. He [husband] died. I lived [at home] without him. I couldn't help thinking about going to join him.' Similarly, C34 said, 'He was a good friend to me. After he died, I felt blue.' 


\section{Loneliness}

Five participants expressed feeling terrible loneliness and stress. As C69 said, 'I was afraid of being alone. Loneliness is a big issue. My sons married and moved out. I lived alone in a big house. Four bedrooms but all empty. I felt stressed and afraid my health would get worse.' Similarly, C73 said, 'I felt alone. All of them [extended family] went out to work during the day. I stayed at home alone. I'm afraid of being alone.'

\section{Reasons for not executing suicide}

Participants' reasons for not actually executing suicide were related to six themes: family members' and friends' support (62.5\%), receiving treatments (58.3\%), finding a way to shift their attention (33.3\%), fear of increasing pressure on one's children (29.2\%), religious beliefs (20.8\%), and not knowing how to execute suicide (20.8\%). Among the participants, $25 \%$ expressed only have one of the six reasons, $50 \%$ expressed two of the six reasons, and $25 \%$ expressed three of the six reasons.

\section{Family members' and friends' support}

Eleven participants expressed their family members' support as the main reason for them not actually executing suicide. As C42 said, 'My wife supports me and wants me to live. I would like to live longer with her.' Similarly, C43 said, 'All of my family members, such as my husband, children, and grandchildren, take care of me. My family members assured me that they need me and cannot lose me. All of them give me the strength to keep going.' Four participants expressed that both family members' and friends' support were important. As C38 said, 'My friends persuaded me to give up those ideas [about suicide]. My son and daughter-in-law also encouraged me to ignore all the bad things.'

\section{Receiving treatments}

Taking psychiatric medications made ten participants felt better and blocked their suicidal ideas. As C3 said, 'Taking the medicine made me not feel so sad. Therefore, I don't have such thoughts [about suicide].' Similarly, C28 said, 'I went to see the psychiatrist. She prescribed medicine and asked me to come back every week. Now, I see her every other week. I feel that I am getting better.' Four participants also had other diseases. The treatments also made them feel better. As C5 said, 'I started going to rehabilitation for my stroke. I considered it [rehabilitation] a chance to go outside and exercise. It's good for my health.'

\section{Finding a way to shift their attention}

Eight participants said that they found things to do to shift their attention from [suicidal] thoughts, even though it might have only partially worked. As C34 said, 'I would keep myself busy to forget the thing [a close friend's death]. However, when I finished my tasks, it [the sad thoughts] may come back.' Similarly, C63 said, 'I watched TV all day until I couldn't keep my eyes open. Then I fell asleep and didn't know anything.'

\section{Fear of increasing pressure on one's children}

Seven participants perceived that suicide would have negative effects on their children. As C58 said, 'After I was sick, I always bothered my son. I had many sleeping pills. I had thoughts about taking all of them. However, I was afraid. If I didn't die, I would add more pressure to my son's life.' Similarly, C42 said, 'I just lost my husband and my son just lost his father. Both of us are suffering. I won't put more pressure on him.'

\section{Religious beliefs}

Five participants expressed that religious beliefs, especially reciting scriptures was important for them. As C65 said, 'When I'm having bad [suicidal] thoughts, I start reciting scriptures. It makes me calm down and not have any bad ideas.' Similarly, C29 said, 'I pray before I go to bed. It makes me feel at peace and gives me the strength to deal with my daily life.'

\section{Not knowing how to execute suicide}

Five participants expressed they did not know how to execute suicide. As C19 said, 'I have ideas [about suicide]. However, I don't know how to do it. Therefore, I don't execute it.' Similarly, C14 said, 'I don't know how to go about doing it [commit suicide].'

\section{Discussion}

This study contributes to suicide studies by describing triggers of suicidal ideation and protective factors against executing suicide as reported by older psychiatric outpatients within a year of their first suicidal ideation. The majority of our participants were diagnosed with depressive disorder, as previously reported [2,3]. However, most of them made their first psychiatric appointment as recommended by their family members rather than by physician referral. This finding highlights an important lesson. Referral systems are well-established in the health care systems of many western societies. However, in many Asian countries people are free to choose to visit hospitals and physicians based on their preferences. Thus, educating Asian family members about suicide is as urgent a task as advocating depression screening. It is important that families with elderly relatives are aware of the risk factors for suicide and bring their depressed relatives as soon as possible to see a psychiatrist for treatment.

Our participants' reasons for suicidal ideation included illness and physical discomfort, conflicts with family members/friends, illness of family members, death of family members/friends, and loneliness. Each reason has been 
reported, but not together, in suicide studies on older adults $[4,6,7,9,15,18,31]$. However, most of these factors (e.g. illness and physical discomfort, conflicts with family members/friends, illness of family members, and death of family members/friends) are not controllable and commonly occur in old age. Feeling lonely is also commonly experienced by older adults but may be relieved by extending social networks and connections $[4,32]$. The most helpful intervention to decrease loneliness found in a meta-analysis was to reduce maladaptive social cognition [33]. Therefore, targeting maladaptive social cognition and increasing opportunities for social interaction may be needed to decrease the prevalence of suicidal ideation [5].

Many of our participants reported developing suicidal ideation after a sudden traumatic life event that they felt they could not change or control. This finding is similar to qualitative findings that older Dutch people's wish to die was either triggered suddenly after traumatic life events or developed gradually after a life full of adversity [10].

Our participants did not mention other risk factors for suicidal ideation reported by older adults, such as financial problems [5,6] and perceived burden to others [9]. Financial problems are a sensitive issue for Taiwanese people who generally prefer not to share information about their income and living expenses with others. Another possible reason for not mentioning financial problems is that most of our participants visited the clinic accompanied by their adult children or other relatives. These relatives may have been contributing to participants' income and/or expenses. Perceived burden to others may not have been expressed by participants because of the value of filial piety in Asian culture, which means that family members expect to take care of each other and to be taken care of when they are in need [34]. Another reason for not mentioning perceived burden to others as a trigger for suicidal thoughts may have been that our participants considered family members' and friends' support as a protective factor against completing suicide.

Our participants considered their protective factors against executing suicide to be family members' and friends' support, receiving treatments, finding a way to shift their attention from suicidal thoughts, fear of increasing pressure on one's children, religious beliefs, and not knowing how to execute suicide. Social support has been well-documented as a protective factor against suicide [16,32]. Among our participants, emotional support was more important than other kinds of social support, similar to a recent report of suicidal ideation in adult African Americans and Caribbean blacks [35]. The benefits of taking anti-depressant medications are recognised [36]. Receiving treatment for painful physical conditions also made participants feel better, which has been found to protect against suicide [37]. In addition, our participants found a way to shift their attention from suicidal thoughts. Shifting attention by engaging in distracting activities is a common coping strategy for older people feeling distress [38]. However, this strategy may only be partially effective.

Our participants also identified fear of increasing pressure on their children as a protective factor against suicide. This finding contradicts a report that suicidal ideation was positively associated with perceived burdensomeness to family members [9]. In other words, older adults in western culture who perceived they were a burden to their families had more suicidal ideation. The difference in these results may be due to cultural factors. Chinese adults are expected to care for their old parents, and our participants were concerned that if they failed in their suicide attempt, their children would have an even greater burden in caring for them.

Religious beliefs were another reason for our older participants to not kill themselves. Similarly, other Asian countries with a strong religious identity were shown to have lower suicide rates, e.g. Thailand and the Philippines where Buddhism and Catholicism, respectively, are widely practised [39]. Our participants reported that religious beliefs provided them positive energy in the form of strength and inner peace, which helped them to deal with problems in their daily life. The role of religion in protecting against older adults' suicidality has been attributed to the social connection of attending religious activities [40]. However, our participants tended to practice their religion alone. This form of religious practice may have been due to their being sick and too weak to go outside. Our participants also identified not knowing how to execute suicide as a protective factor against executing suicide. Patients in this situation and those who seek protection against suicidal ideation by shifting their attention may benefit from antidepressant treatment. Another important approach for treating patients with suicide problems, as mentioned before, is cognitive therapy to address maladaptive social cognition [5].

The experiences of older people with suicidal ideation or a wish to die have not been widely explored. One interview study of older Dutch people with at least a weak wish to die found that they perceived developing thoughts about death as a positive thing or as a release from problems [10]. They perceived dying as a way to reclaim control when they could not manage problems, were reluctant to ask other people to help, and were not convinced that these options would make much difference. They started to consider the negative and positive aspects of both living and dying. After weighing these four dynamic aspects, older Dutch participants formed a balance of feelings toward living and dying [10]. As 
mentioned before, most of our participants developed suicidal ideation after sudden traumatic life events. However, these suicidal thoughts were disrupted or diminished by support from their family members and friends or by receiving treatments that relieved their chronic illness or depressive symptoms.

Since many people in Asian countries can freely choose hospitals and physicians based on their preferences, psychiatrists in busy clinics may be challenged to assess new onset cases without any previous medical record. Moreover, Asians have difficulty discussing death and are afraid of visiting psychiatric clinics because of the social stigmatisation of psychiatric patients [41]. Understanding the triggers of suicide ideation identified by our participants may help clinical psychiatrists open a channel for conversation and more readily make their diagnosis. Understanding the protective factors against executing suicide identified by our participants may help psychiatrists not only treat depression, but also enhance protective factors.

\section{Limitations}

This study had two limitations. The sample was recruited by convenience and only from two hospitals in northern Taiwan. As a result, our sample may have consisted of more females, more young-old adults, and fewer single older people, even though the distributions are similar to those of older people in Taiwan [42]. Further studies may consider expanding data collection settings and using purposeful sampling to obtain a study sample with more even proportions of each demographic characteristic.

\section{Conclusions}

In this qualitative descriptive study, triggers of suicide ideation related by older psychiatric outpatients with a year of their initial suicide ideation were related to five major themes: illness and physical discomfort, conflicts with family members/friends, illness of family members, death of family members/friends, and loneliness. We also identified six major themes related to their protective factors against executing suicide: family members' and friends' support, receiving treatments, finding a way to shift their attention, fear of increasing pressure on one's children, religious beliefs, and not knowing how to execute suicide. The triggers of suicide ideation are similar to those in western studies. Targeting maladaptive social cognition and increasing opportunities for social interaction may be needed to decrease older adults' loneliness and the prevalence of suicidal ideation. Furthermore, suicide prevention programmes should educate family members of older depressed people about suicide and suicidal ideation. Finally, older people with suicide ideation should receive treatments, social support (especially family members' and friends' emotional support), be encouraged to practice their religious beliefs, and receive cognitive therapy to prevent them from executing suicide.

\section{Competing interests}

The authors declare that they have no competing interest.

\section{Authors' contributions}

SH Lee assisted with designing the study, collecting and analysing the data, and writing the manuscript. YF Tsai designed the study, supervised the data collection, analysed data and wrote the manuscript. YJ Chen and LB Hwang assisted with collecting and analysing the data, and writing the manuscript. All authors read and approved the final manuscript.

\section{Acknowledgements}

This study was funded by the National Science Council, an agency of the Taiwan government (NSC 100-2314-B-182-018), and Chang Gung Memorial Hospital (CMRPD1B0491, CMRPD1B0492), Yun-Fang Tsai, PI.

\section{Author details}

${ }^{1}$ Department of Psychiatry, Chang Gung Memorial Hospital at Linkou, 5 Fusing Street, Kwei-Shan, Tao-Yuan, Taiwan. ${ }^{2}$ College of Medicine, Chang Gung University, 259 Wen-Hwa 1st Road, Kwei-Shan, Tao-Yuan, Taiwan. ${ }^{3}$ School of Nursing, College of Medicine, Chang Gung University, 259 Wen-Hwa 1st Road, Kwei-Shan, Tao-Yuan, Taiwan. ${ }^{4}$ Department of Nursing, Chang Gung Memorial Hospital at Keelung, 222 Maijin Road, Keelung, Taiwan. ${ }^{5}$ Department of Nursing, Chang Gung Memorial Hospital at Linkou, 5 Fusing Street, Kwei-Shan, Tao-Yuan, Taiwan.

Received: 21 May 2014 Accepted: 16 September 2014

Published online: 18 November 2014

\section{References}

1. World Health Organization: Public Health Action for the Prevention of Suicide: A Framework. Geneva: World Health Organization; 2012

2. O'Connell $H$, Chin AV, Cunningham $C$, Lawlor BA: Recent developments: suicide in older people. BMJ 2004, 329:895-899.

3. Conwell $Y$, Thompson C: Suicidal behavior in elders. Psychiatr Clin North Am 2008, 31:333-356.

4. Fässberg MM, van Orden KA, Duberstein P, Erlangsen A, Lapierre $S$, Bodner E, Canetto SS, De Leo D, Szanto K, Waern M: A systematic review of social factors and suicidal behavior in older adulthood. Int J Environ Res Public Health 2012, 9:722-745.

5. Almeida OP, Draper B, Snowdon J, Lautenschlager NT, Pirkis J, Byrne G, Sim M Stocks N, Flicker L, Pfaff JJ: Factors associated with suicidal thoughts in a large community study of older adults. Br J Psychiatry 2012, 201:466-472.

6. Rurup ML, Deeg DJ, Poppelaars JL, Kerkhof AJ, Onwuteaka-Philipsen BD: Wishes to die in older people: a quantitative study of prevalence and associated factors. Crisis 2011, 32:204-216.

7. Yip PS, Chi I, Chiu H, Chi Wai K, Conwell Y, Caine E: A prevalence study of suicide ideation among older adults in Hong Kong SAR. Int J Geriatr Psychiatry 2003, 18:1056-1062.

8. Nadorff MR, Fiske A, Sperry JA, Petts R, Gregg JJ: Insomnia symptoms, nightmares, and suicidal ideation in older adults. J Gerontol B Psychol SCi Soc Sci 2013, 68:145-152

9. Cukrowicz KC, Cheavens JS, Van Orden KA, Ragain RM, Cook RL: Perceived burdensomeness and suicide ideation in older adults. Psychol Aging 2011, 26:331-338.

10. Rurup ML, Pasman HR, Goedhart J, Deeg DJ, Kerkhof AJ, OnwuteakaPhilipsen BD: Understanding why older people develop a wish to die: a qualitative interview study. Crisis 2011, 32:204-216.

11. Bedrosian RC, Beck AT: Cognitive aspects of suicidal behavior. Suicide Life Threat Behav 1979, 9:87-96.

12. Kissane M, McLaren S: Sense of belonging as a predictor of reasons for living in older adults. Death Stud 2006, 30:243-258.

13. McLaren S, Gomez R, Bailey M, Van Der Horst RK: The association of depression and sense of belonging with suicidal ideation among older adults: applicability of resiliency models. Suicide Life Threat Behav 2007, 37:89-102.

14. Rowe $J$, Conwell Y, Schulberg HC, Bruce ML: Social support and suicidal ideation in older adults using home healthcare services. Am J Geriatr Psychiatry 2006, 14:758-766. 
15. Ku YC, Tsai YF, Lin YC, Lin YP: Suicide experiences among institutionalized older veterans in Taiwan. Gerontologist 2009, 49:746-754.

16. Wang YW, Tsai YF, Wong TK, Ku YC: Development and psychometric testing of a Chinese-language instrument for assessing institutionalised older males' motivations for living. J Clin Nurs 2013, 22:2867-2875.

17. Chiu HF, Dai J, Xiang YT, Chan SS, Leung T, Yu X, Hou ZJ, Ungvari GS, Caine ED: Suicidal thoughts and behaviors in older adults in rural China: a preliminary study. Int J Geriatr Psychiatry 2012, 27:1124-1130.

18. Chen YJ, Tsai YF, Ku YC, Lee SH, Lee HL: Perceived reasons for, opinions about, and suggestions for elders considering suicide: elderly outpatients' perspectives. Aging Ment Health 2014, 18:593-599.

19. Kim S, Ha JH, Yu J, Park DH, Ryu SH: Path analysis of suicide ideation in older people. Int Psychogeriatr 2014, 26:509-515.

20. Alexopoulos GS, Bruce ML, Hull J, Sirey JA, Kakuma T: Clinical determinants of suicidal ideation and behavior in geriatric depression. Arch Gen Psychiatry 1999, 56:1048-1053.

21. Heisel MJ, Duberstein PR, Conner KR, Franus N, Beckman A, Conwell Y: Personality and reports of suicide ideation among depressed adults 50 years of age or older. J Affect Disord 2006, 90:175-180.

22. Britton PC, Duberstein PR, Conner KR, Heisel MJ, Hirsch JK, Conwell Y: Reasons for living, hopelessness, and suicide ideation among depressed adults 50 years or older. Am J Geriatr Psychiatry 2008, 16:736-741.

23. Kasckow J, Montross L, Golshan S, Mohamed S, Patterson T, Sollanzano E, Zisook S: Suicidality in middle aged and older patients with schizophrenia and depressive symptoms: relationship to functioning and Quality of Life. Int J Geriatr Psychiatry 2007, 22:1223-1228.

24. Purcell B, Heisel MJ, Speice J, Franus N, Conwell Y, Duberstein PR: Family connectedness moderates the association between living alone and suicide ideation in a clinical sample of adults 50 years and older. Am J Geriatr Psychiatry 2012, 20:717-723.

25. Kuo CJ, Gunnell D, Chen CC, Yip PS, Chen YY: Suicide and non-suicide mortality after self-harm in Taipei City, Taiwan. Br J Psychiatry 2012, 200:405-411.

26. Yip PK, Shyu YI, Lin SI, Lee JY, Chou CF, Chen RC: An epidemiological survey of dementia among elderly in an urban district of Taipei. Acta Neurol Sin 1992, 1:347-354.

27. Sheehan DV, Lecrubier $Y$, Sheehan KH, Amorim P, Janavs J, Weiller $E_{1}$ Hergueta T, Baker R, Dunbar GC: The Mini-International Neuropsychiatric Interview (M.I.N.I.): the development and validation of a structured diagnostic psychiatric interview for DSM-IV and ICD-10. J Clin Psychiatry 1998, 59(Suppl 20):22-33.

28. Denzin NK, Lincoln YS: Handbook of Qualitative Research. Beverly Hills, CA: Sage; 1994.

29. Lincoln YS, Guba EG: Naturalistic Inquiry. Beverly Hills, CA: Sage; 1985

30. Burke WJ, Rocaforte WH, Wengel SP: The short form of the Geriatric Depression Scale: a comparison with the 30-item form. J Geriatr Psychiatry Neurol 1991, 4:173-178.

31. Jahn DR, Poindexter EK, Graham RD, Cukrowicz KC: The moderating effect of the negative impact of recent life events on the relation between intrinsic religiosity and death ideation in older adults. Suicide Life Threat Behav 2012, 42:589-601.

32. Van Orden K, Conwell Y: Suicides in late life. Curr Psychiatry Rep 2011, $13: 234-241$

33. Masi CM, Chen HY, Hawkley LC, Cacioppo JT: A meta-analysis of interventions to reduce loneliness. Pers Soc Psychol Rev 2011, 15:219-266.

34. Dai YT, Dimond MF, Filial piety: A cross-cultural comparison and its implications for the well-being of older parents. J Gerontol Nurs 1998, 24:13-18.

35. Lincoln KD, Taylor RJ, Chatters LM, Joe S: Suicide, negative interaction and emotional support among black Americans. Soc Psychiatry Psychiatr Epidemiol 2012, 47:1947-1958.

36. Erlangsen A, Conwell Y: Age-related response to redeemed antidepressants measured by completed suicide in older adults: a nationwide cohort study. Am J Geriatr Psychiatry 2014, 22:25-33.

37. Tsai YF, Wong TK, Ku YC, Liu WC: Reasons for living among older male Chinese residents of veterans' homes. J Adv Nurs 2012, 68:1978-1987.

38. Tsai YF, Liu LL, Tsai HH, Chung SC: Self-care management and risk factors for depressive symptoms among elderly outpatients in Taiwan. Int Psychogeriatr 2012, 24:278-287.

39. Chen YY, Wu KC, Yousuf S, Yip PS: Suicide in Asia: opportunities and challenges. Epidemiol Rev 2012, 34:129-144.
40. Rushing NC, Corsentino E, Hames $L$, Sachs-Ericsson N, Steffens DC: The relationship of religious involvement indicators and social support to current and past suicidality among depressed older adults. Aging Ment Health 2013, 17:366-374

41. Lauber C, Rössler W: Stigma towards people with mental illness in developing countries in Asia. Int Rev Psychiatry 2007, 19:157-178.

42. Department of Statistics, Ministry of Interior: Structures of population in 2013. http://sowf.moi.gov.tw/stat/week/week10303.doc

doi:10.1186/s12888-014-0269-9

Cite this article as: Lee et al:: Triggers of suicide ideation and protective factors of actually executing suicide among first onset cases in older psychiatric outpatients: a qualitative study. BMC Psychiatry 2014 14:269.

\section{Submit your next manuscript to BioMed Central and take full advantage of:}

- Convenient online submission

- Thorough peer review

- No space constraints or color figure charges

- Immediate publication on acceptance

- Inclusion in PubMed, CAS, Scopus and Google Scholar

- Research which is freely available for redistribution

Submit your manuscript at www.biomedcentral.com/submit
C Biomed Central 